${ }^{1}$ Aintree University Hospital, Liverpool \&

2Betsi Cadwaladr Univesity Health Board, Bangor

UK

\title{
Patient Generated Index in HN Chemosensory \& auditory complaints: An observational study
}

\section{Usama F Kamel1 \& Ushan Andrady²}

Appendix A PGI Patient Generated Index of Quality of Life

Your answers to the following steps will tell us how your life is affected by your Smell, Taste and Hearing senses.

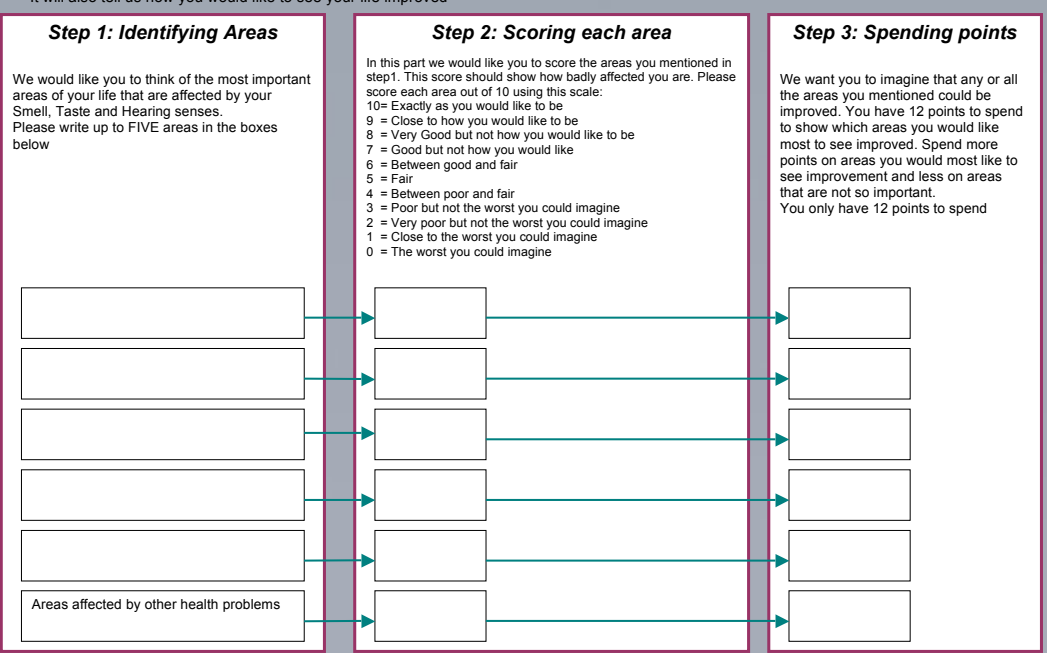

PGI

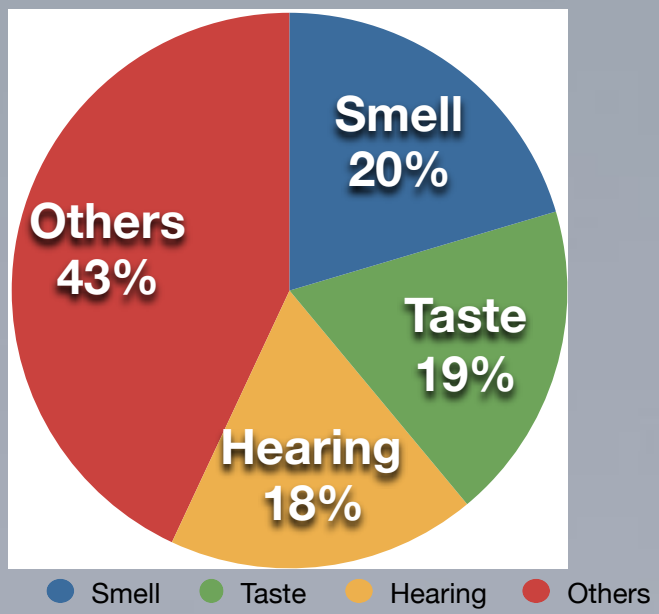

HIV is a chronic manageable disease. The disease and/or antiretroviral therapy treatment may lead to patient complaints regarding their hearing and chemosensory perception. Patient Generated Index (PGI) has been a validated patient generated questionnaire, which is flexible to capture areas relevant to patients. It has not been used before to assess the chemosensory (smell and taste) and auditory concerns of HIV positive patients. Methods: PGI questionnaire was distributed to nineteen HIV patients attending the research clinic. Patients had five areas to record their perception in relation to senses (smell, taste and hearing). They then score how do they feel about this particular area, then they spend points to improve the area when needed. Patients were recruited from a HIV clinic after giving their informed consent matching inclusion/exclusion criteria. The questionnaire had five sections where patients identify problems matter to them in relation to smell, taste and hearing. The sixth question is generated by the patient in relation to other health areas of concern to the individual. Results: PGI scores $(n=19)$ mean was 5.39 (range $1.08-8, S D$ 1.93). Smell mean scores 1.1, taste was 1 and hearing was 0.97 Smell was $20.4 \%$ of the total PGI score; taste was $18.6 \%$ and hearing $18 \%$ of the PGI score. Patients scored 2.32 (43\% of the total PGI score) in other areas (back pain, arthritis, stomach pains, ...)

Conclusions: This is an observational cohort study to document the PGI results in relation to auditory and chemosensory perception in HIV positive patients. Although auditory and chemosensory problems in five areas scores altogether $57 \%$ of the PGI score, others (one area question), patients scored $43 \%$ of the total PGI score. other complaints preoccupied patients and were relatively more cumbersome than the three senses.

Ruta et al. A New Approach to the Measurement of Quality of Life: The Patient-Generated Index. Medical Care. 1994 Nov;32(11):1109-1126 